\title{
Fungal cerebellar tonsillar abscess as a cause of quadriparesis
}

Sir,

Fungal infections of the brain manifest as meningitis, granuloma, abscess. Fungal abscess occurring in the cerebellar tonsil has not been reported so far (to our knowledge). The patient presented with progressive quadriparesis due to high cervical cord compression which is uncommon.

A 35-year-old male presented with neck pain and progressive quadriparesis of two months duration. Examination revealed spastic quadriparesis with Grade 3 power with diminished sensation from $\mathrm{C}_{3}$ dermatome downwards and bladder dysfunction. Optic fundi were normal. There were no cerebellar signs. No foci of sepsis could be made out. The MRI brain showed an oval-shaped well-defined mass which was hypointense in $\mathrm{T}_{1}$ weighted image [Figure 1] and hyperintense in $\mathrm{T}_{2}$ weighted image occupying the region of midline cerebellum compressing the cervico-medullary junction [Figure 2].

Suboccipital craniectomy and removal of posterior arch of $\mathrm{C}_{1}$ was done and the mass lesion occupying the right cerebellar tonsil was exposed. Aspiration of the mass revealed purulent material. The abscess was thick-walled and was excised in toto. Histopathological examination revealed septate filaments showing acute angled branches in the background of necrotic material, suggestive of Aspergillus mycelia [Figure 3]. Pus was cultured in Sabouraud Dextrose agar and there was no growth till two weeks. Subsequent investigations did not reveal any immunocompromised state.

Patient was put on Tab. Itraconazole $400 \mathrm{mg} /$ day orally for three months and was maintained on $200 \mathrm{mg} /$ day

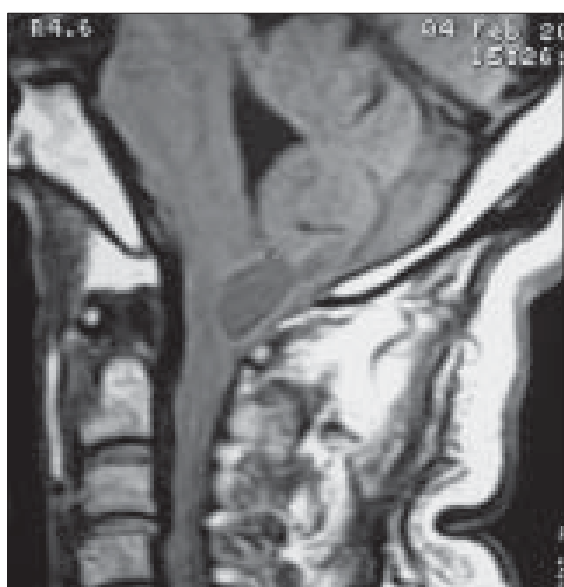

Figure 1: T1 hypointense area surrounded by hyperintense rim at CV junction 


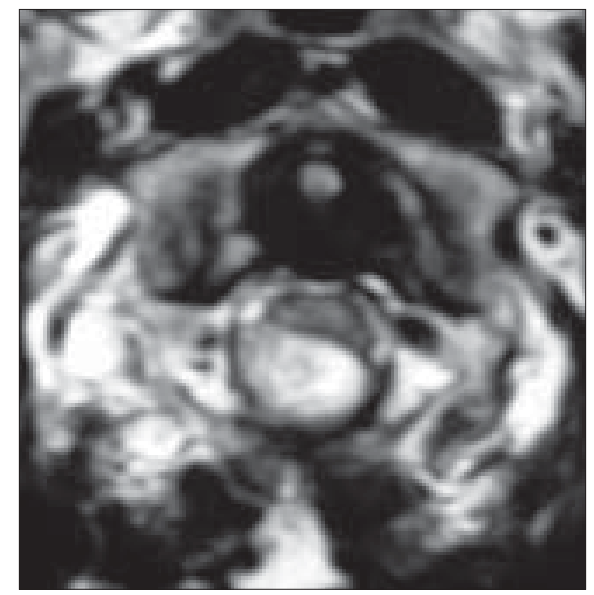

Figure 2: T2 Axial section at C1 level showing a hyperintense sol compressing spinalcord



Figure 3: Fungal mycelium with necrotic background seen in PAS staining in $\times 200$

from the fourth to sixth month. The antifungal treatment lasted for six months. He is under our follow-up for the past one year and he made complete neurological recovery.

Fungi are common in the environment, but only a few are pathogenic. In general, fungi are organisms of low pathogenicity, emerging as opportunistic organisms thriving in a compromised host, however, some will infect even normal hosts. ${ }^{[1-3]}$ In the nervous system, the infection can be found in the cerebral parenchyma, the meninges or the vascular system. ${ }^{[1]}$

Fungal infection as an etiology of brain abscess is common in immunosuppressed patients either due to systemic illness or due to drugs. ${ }^{[4]}$ Aspergillus fumigatus is the most common human pathogen in the genus Aspergillus. It has branching septate hyphae varying from 4-12 $\mathrm{mm}$ in width, which show dichotomous branching and produce numerous spores on the tips of long conidiophores. ${ }^{[1]}$

Aspergillosis is diagnosed on direct examinations and culture, however, the diagnosis of aspergillosis of the central nervous system (CNS) is difficult. In purulent lesions, pus is seen in the center of the abscesses with abundant polymorphs at the periphery. Granulomas consist of lymphocytes, plasma cells and fungal hyphae. ${ }^{[1]}$

Aspergillus is cultured optimally on Sabouraud's agar and demonstrates characteristic conidiophores. However, blood and cerebrospinal fluid cultures, even in disseminated disease, are frequently negative. ${ }^{[1]}$

Fungal abscess can be managed medically by antifungal drugs provided the organism is known, size of the abscess is small and source of the infection is located. Liposomal amphotericin has been demonstrated to be safe and efficacious in the management of CNS aspergilloma. ${ }^{[1]}$ If the abscess is not amenable to medical management stereotactic aspiration or excision of abscess is the treatment of choice ${ }^{[4]}$ Radical surgical debridement can be curative in Aspergillus brain abscess if the extent of resection extends into uninvolved tissue. ${ }^{[5]}$

This is the first report of cerebellar tonsillar abscess due to fungal etiology.

\section{Kodeeswaran Marappan, K. Deiveegan, D. Balasubramanian, A. Sundaram}

Department of Neurosurgery and Pathology, Govt Stanley Medical College, Chennai - 600 001, India. E-mail: neurokodee@gmail.com

\section{References}

1. Nadkarni T, Goel A. Aspergilloma of the brain: An overview. J Postgrad Med 2005;51:37-41.

2. Haran RP, Chandy MJ. Intracranial Aspergillus granuloma. Br J Neurosurg 1993;7:383-8.

3. Kawakami N, Nishizaki T, Sugiyama S, Ito H. Aspergillus brain abscess in a patient with normal immunity: Case report. Neurol Med Chir (Tokyo) 1994;34:237-40.

4. Loftus CM, Osenbach RK, Biller J. Diagnosis and management of brain abscess. In: Neurosurgery. $2^{\text {nd }}$ ed. Wilkins RH, Rangachary SS. McGraw Hill: USA; 1996. p. 3285-98

5. Green M, Wald ER, Tzakis A, Todo S, Starzl TE. Aspergillosis of the CNS in a pediatric liver transplant recipient: Case report and review. Rev Infect Dis 1991;13:653-7.

Accepted on 12-02-2007 